\title{
Influencia de las Tecnologías de Información y Comunicación en los roles e interrelaciones entre estudiantes y docentes en programas presenciales de educación superior*
}

\author{
Norma Julieth Ruiz Rodríguez ${ }^{* *}$, Martha Ruth Mendoza Torres**, Luis Gabriel Ferrer ${ }^{* * * *}$
}

\section{Resumen}

Recibido: 8 de junio de 2014

Evaluado: 14 de julio de 2014

Aceptado: 22 de agosto de 2014
El propósito de este artículo es hacer una reflexión dirigida a directivas y docentes de instituciones de educación superior, acerca de cómo el uso de las Tecnologías de Información y Comunicación (TIC) influye en los roles e interrelaciones entre estudiantes y docentes, en los procesos de enseñanza aprendizaje en programas presenciales de ese nivel. De esta se concluye que cuando las TIC se utilizan como apoyo a la labor educativa de una forma planeada, emerge una dinámica de cambio en la interrelación entre estudiantes y docentes, donde los primeros asumen un papel activo en la construcción de su aprendizaje individual y colectivo, en tanto que los docentes se convierten en arquitectos y gestores de ambientes y experiencias de aprendizaje mediadas por TIC, y guías en el proceso de búsqueda y análisis de información en el mundo digital, además de orientadores del aprendizaje; diferente a la educación tradicional donde el docente es visto como poseedor del conocimiento y el estudiante como receptor pasivo del mismo.

Artículo de revisión de tema. Derivado de la investigación "Diseño, implementación y evaluación de estrategias de enseñanza-aprendizaje basadas en Tecnologías de la Información y la Comunicación para los programas de Postgrado de Seguridad de la Facultad de Relaciones Internacionales, Estrategia y seguridad", proyecto EES-1228 de la Universidad Militar Nueva Granada, Bogotá, Colombia. Cómo citar este artículo: Ruiz Rodríguez, N. J., Mendoza Torres, M. R. y Ferrer, L.G. (2014). Influencia de las Tecnologías de Información y Comunicación en los roles e interrelaciones entre estudiantes y docentes en programas presenciales de Educación Superior. Hallazgos, 11(22), 435-454.

** Magíster en Educación. Magister en Informática Educativa. Integrante del grupo de investigación Estudios Internacionales y Políticos (Colombia).. E-mail: norma.ruiz@unimilitar.edu.co

*** Especialista en Docencia Universitaria. Candidata a doctor en Educación (Colombia). E-mail: mendozamartha@unbosque.edu.co

**** Economista, Especialista en Pedagogía de la Virtualidad. Integrante del grupo de investigación Estudios Internacionales y Políticos (Colombia). E-mail: luis.ferrer@unimilitar.edu.co 


\section{The influence of ICTs on the roles and inter-relations among students and professors in higher Education in-campus programs}

Este cambio de dinámica en los procesos de aprendizaje propiciado por el uso de las TIC promueve el aprendizaje significativo a nivel individual y el trabajo colaborativo y aprendizaje en red, como expresión de la construcción social del aprendizaje que se da en la interacción entre estudiantes y docentes como parte del colectivo, lo cual nos invita a repensar tanto el modelo educativo de las instituciones de educación superior, como la formación de los docentes.

Palabras clave: Tecnologías de Información y Comunicación, interrelación estudiante y docente, estrategias de enseñanza y aprendizaje, aprendizaje significativo, trabajo colaborativo. 


\section{Abstract}

The purpose of this article is to make a reflection aimed at managers and teachers of institutions of higher education, about how the use of ICT influences the roles and interrelationships between students and teachers, in the processes of teaching and learning in face-to-face programs at that level.

From this, it is concluded that when ICTs are used as support for the educational work in a planned way, emerges a dynamic of change in the relationship between students and teachers, where the first assume a role both individual and collective, active in the construction of their learning while teachers become architects and managers of environments and ICT-mediated learning experiences, and guides the process of search and analysis of information in the digital world, in addition to guiding of learning, different from the traditional education where the teacher is seen as holder of the knowledge and the student as a passive recipient of the same.

This change in the dynamic process of learning by the use of ICT, promotes meaningful learning at the individual level, and collaborative work and learning in network, as an expression of the social construction of the learning that occurs in the interaction between students and teachers as part of the collective, which invites us to rethink the educational model of the institutions of higher education and teacher training.

Keywords: Technologies of Information and Communication, student and teacher interaction, teaching and learning, significant learning, collaborative work. 


\section{Influência das Tecnologias de Informação e Comunicação nos papéis e interrelações entre alunos e professores em programas de sala de aula do Ensino Superior}

\section{RESUMO}

O objetivo deste artigo é fazer uma reflexão destinada às diretrizes e aos professores de instituições de ensino superior, sobre como o uso das Tecnologias de Informação e Comunicação (TIC) influenciam nos papéis e interrelações entre alunos e professores, nos processos de ensino e aprendizagem em programas de sala de aula desse nível. A partir disso conclui-se que quando as TIC são utilizadas para apoiar o trabalho educativo de forma planejada, emerge uma dinâmica de cambio na interrelação entre alunos e professores, onde os primeiros assumem um papel ativo na construção de sua aprendizagem individual e coletiva, enquanto que os professores se tornam em arquitetos e gestores de ambientes e experiências de aprendizagem mediada pelo TIC, e guias no processo de procura e análise de informação no mundo digital, além de mentores de aprendizagem; diferente à educação tradicional onde o professor é visto como possuidor do conhecimento e o aluno como receptor passivo desse conhecimento.

Esta mudança na dinâmica nos processos de aprendizagem facilitada pelo uso das TIC promove a aprendizagem significativa ao nível individual e o trabalho colaborativo e aprendizagem na rede e, como uma expressão da construção social da aprendizagem que ocorre na interação entre alunos e professores como parte do coletivo, que nos convida a repensar tanto o modelo educacional das instituições de ensino superior, como a formação dos professores.

Palavras-chave: Tecnologias d Informação e Comunicação, interrelação aluno e professor, estratégias de ensino e aprendizagem, aprendizagem significativa, trabalho colaborativo. 


\section{INTRODUCCIÓN}

La integración de Tecnologías de Información y Comunicación (TIC), como medios de apoyo a los procesos de enseñanza-aprendizaje en educación superior, abarca más de dos décadas y ha dependido de factores endógenos y exógenos a las instituciones educativas, los primeros como consecuencia del tiempo que ha tomado el avance de las mismas y los segundos de la forma como estas tecnologías se han ido integrando a los espacios personales, laborales y organizacionales. Esto ha dado como resultado el emerger de una sociedad basada en el acceso y uso de la información, con el objetivo de convertirla en conocimiento útil (Unesco, 2008), sociedad que a su vez demanda de la educación superior la formación de profesionales con competencias en el uso y aplicación de estas tecnologías, lo cual los habilitará para desempeñarse adecuadamente en este nuevo entorno.

Las TIC son consideradas hoy en día como herramientas de gestión del conocimiento que mejoran el aprendizaje y lo hacen significativo para los estudiantes, por cuanto facilitan el intercambio de información científica, permiten el acceso a contenidos lingüísticos y culturales diversos (Unesco, 2010) y facilitan la colaboración y comunicación sincrónica y asincrónica entre estudiantes y docentes, en ambientes de aprendizaje abundantes en fuentes de información que permiten explorar, observar y analizar multiplicidad de fenómenos y situaciones, aspecto que facilita la construcción de conocimientos a través del estímulo de la comprensión conceptual, la flexibilidad mental, la creatividad y la innovación, y conduce así a los estudiantes a convertirse en constructores de su propio conocimiento.
Ante este nuevo panorama, se hace necesario revisar cómo el uso de las TIC en los procesos de enseñanza y aprendizaje influye en la dinámica de las interrelaciones entre estudiantes y docentes, por cuanto el uso de las mismas abre nuevas posibilidades para estimular el aprendizaje significativo y el trabajo colaborativo entre pares, pero también implica cambios en los modos de interacción entre estos protagonistas de los procesos de enseñanza aprendizaje.

Por esto, el propósito de este artículo es hacer una reflexión, dirigida a directivas y docentes de instituciones de educación superior, acerca de cómo influyen las TIC en los roles e interrelaciones entre estudiantes y docentes en los procesos de enseñanzaaprendizaje en programas presenciales de educación superior, que conduzcan a repensar tanto el modelo educativo de las instituciones de educación superior, como la formación de los docentes.

Como consecuencia, la primera parte de este artículo presenta un breve marco teórico sobre aprendizaje significativo, aprendizaje y trabajo colaborativo y constructivismo social, por cuanto se espera que estos tipos de aprendizaje sean estimulados como consecuencia de la aplicación de las TIC en actividades donde se da la interacción entre estudiantes y docentes, las cuales combinan los conocimientos individuales según el tema tratado con los saberes, recursos y estrategias docentes. La segunda parte hace una reflexión acerca de la situación del docente frente al reto que representa la integración de estas tecnologías a las actividades de enseñanza y aprendizaje y de qué manera se entrelaza con las instituciones de educación 
superior. En la tercera parte se identifican cambios detectados de roles e interrelaciones entre estudiantes y docentes, derivados del uso de tecnologías de información y comunicación en las actividades de aprendizaje. A continuación, se presentan a modo de ilustración dos ejemplos de cómo se articulan las estrategias de enseñanza aprendizaje con las TIC como apoyo y se concluye con las reflexiones finales de los autores.

\section{Las TeCNOlogías de INFORMACIÓN Y COMUNICACIÓN, Y EL APRENDIZAJE SIGNIFICATIVO, APRENDIZAJE Y TRABAJO
COLABORATIVO}

Dado que el uso de las TIC en educación demanda del estudiante la búsqueda, análisis y comparación de información con sus conocimientos de referencia y la construcción de ideas, con lo que se da un aprendizaje significativo y se fortalece la comunicación con compañeros y docentes, y la construcción grupal de conocimiento, hecho que da lugar al trabajo colaborativo, es importante explicar en qué consisten estos dos conceptos (aprendizaje significativo y trabajo colaborativo).

El aprendizaje significativo se refiere a relacionar nuevos conceptos o información con lo que el estudiante ya sabe (Ausubel, Novak y Hasenian, 1983, p. 37), para lo cual la nueva información debe poder relacionarse con los conocimientos previos del estudiante, y este debe tener disposición hacia el aprendizaje, es decir, interés en aprender o, lo que es lo mismo, una "actitud activa" (Ontoria y Ballesteros, 2011, p. 20) frente al proceso de aprendizaje.
De acuerdo con estas condiciones, este tipo de aprendizaje es de carácter individual, ya que se basa en los conocimientos previos de cada estudiante y la disposición del mismo. Como en la actualidad los estudiantes traen consigo, en mayor o menor grado, conocimientos, saberes y experiencias relacionadas con el uso de las TIC, son estos contenidos los fundamentos que posibilitan el aprendizaje significativo mediante actividades apoyadas en las TIC, que estimulan y motivan el aprendizaje en los estudiantes, para convertirlos en constructores de su propio conocimiento.

En adición a lo anterior, dado que las actividades de aprendizaje que utilizan las TIC propician las interacciones entre los estudiantes y de estos con los docentes, emerge un contexto de constructivismo sociocultural (López Carrasco, 2013, p. 100); enfoque desarrollado por Leo Vigotsky (1896, 1934), el cual argumenta que el aprendizaje es la suma del desarrollo cognitivo individual y el desarrollo social que se da en las interacciones entre grupos de estudiantes y docentes (Antunes, 2009, p. 26). Allí el aprendizaje depende tanto de las estructuras cognitivas de cada estudiante, como de lo que puede aprender a través de las interacciones con compañeros y docentes. Por lo tanto, la construcción del aprendizaje será la suma de procesos individuales más procesos de socialización, donde con el apoyo de todos el estudiante desarrolla su autonomía para la resolución de problemas y la aplicación práctica del conocimiento construido (Antunes, 2009, p. 20). Por esto, el conocimiento y aprendizaje obtenidos en la dinámica de esas interacciones sociales (Cerezo Huertas, 2006, p. 16)son en parteresultado de un aprendizaje colaborativo. Así 
mismo, Johnson y Johnson (1999, p. 263) señalan que el conocimiento es social y se construye a partir de los esfuerzos cooperativos por aprender, entender y resolver problemas. Este postulado tiene relación con el concepto de la zona de desarrollo próximo (ZDP) planteada por Vigotsky, entendida como la distancia entre el nivel de aprendizaje individual de un estudiante y el nivel que puede alcanzar con el apoyo de compañeros más competentes o con mayor conocimiento y experiencia en determinados campos, y bajo la orientación de los docentes (Antunes, 2009, p. 26).

En este sentido, la integración de las TIC a los procesos de enseñanza-aprendizaje brinda a estudiantes y docentes formas y recursos para incrementar la interacción social, lo que genera zonas de desarrollo próximo donde el aprendizaje alcanza mayores niveles de complejidad y profundidad (Antunes, 2009, p. 28). Así los docentes a través de las interacciones traspasan de forma progresiva la responsabilidad del aprendizaje a los estudiantes (Serrano y Pons, 2011, p. 15), lo cual los conduce a una mayor autonomía y estos a través de debates conceptuales y apoyo mutuo construyen un mayor aprendizaje.

Así es como este aprendizaje, propio del modelo pedagógico socioconstructivista, entiende el aprender como una experiencia social, resultado de un proceso de construcción del conocimiento en un contexto social y cultural concreto (Servicios Educativos del Magisterio, 2003, p. 134), donde cada estudiante interactúa con otros conformando redes sociales y comunidades de aprendizaje, donde aprender significa aprender con otros (Márques, 1999).
En esas redes y comunidades, el estudiante interactúa con expertos y pares de manera personal y virtual, lo que le brinda bases de apoyo para su proceso de construcción de conocimiento, aprovechando las ventajas de los esquemas de aprendizaje colaborativo (Márques, 1999), donde cada estudiante construye su aprendizaje según sus conocimientos, esquemas, saberes, experiencias previas y su contexto (Márques, 1999) en la interacción con los otros. En este modelo el docente asume el papel de guía y dinamizador de las redes de aprendizaje y el estudiante se convierte en integrante activo de dichas redes y constructor de su propio conocimiento. Sin embargo, la participación e interacción en redes no garantizan el aprendizaje colaborativo por sí solo, ya que es necesario que el docente promueva en los estudiantes estrategias de aprendizaje, de tal forma que se cree un ambiente estimulante de enseñanza donde los estudiantes estimulen y motiven a sus compañeros para que se impliquen en el aprendizaje (Barkley, Cross y Howell, 2007, p. 37). Para lograr el aprendizaje colaborativo de acuerdo con Johnson y Johnson (1999, p. 245), es necesario contar con cinco elementos básicos: interdependencia positiva, para que los alumnos estén convencidos de que son tan responsables del aprendizaje del otro como del propio; responsabilidad individual, para que cada uno haga su parte del trabajo; interacción cara a cara, donde todos estimulen el aprendizaje de los demás mediante la ayuda y el aliento; uso adecuado de las habilidades interpersonales y en grupos pequeños, necesarias para trabajar juntos con eficacia y procesamiento del trabajo grupal y de cómo se podría mejorar. 
Figura1. Elementos básicos del trabajo colaborativo
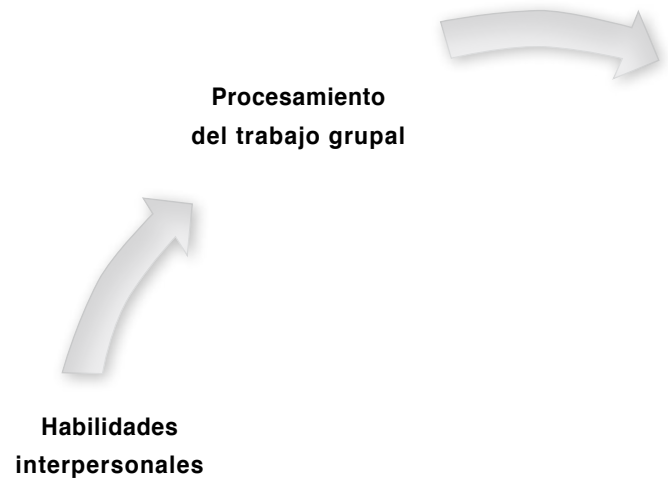

interpersonales

Responsabilidad individual
Interacción

cara a cara
Interdependencia

positiva
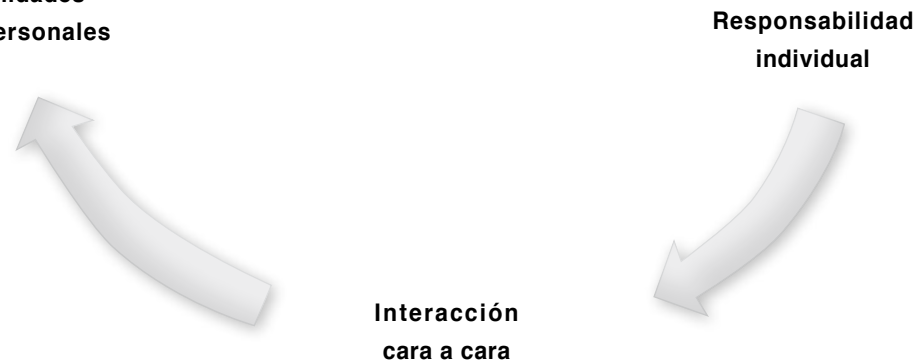

Fuente: Johnson y Johnson (1999)

Así es como este aprendizaje surge a partir de un trabajo colaborativo, definido como "la elaboración conjunta de un producto final, a partir de una combinación de aportaciones individuales y de procesos de debate; mejorando la interacción y el trabajo entre los miembros del grupo, facilitando el intercambio y la distribución del conocimiento, así como la creación de expertos en la comunidad de trabajo" (Tobón, 2007, p. 35). En estos espacios la colaboración se da entre pares y es natural y espontánea (Barkley, Crossy Howell, 2007), lo cual se puede apreciar en las actividades de aprendizaje mediadas por TIC, donde se da la comunicación oral y escrita entre los estudiantes y la conformación de pequeños grupos de trabajo organizados, sea por iniciativa de los estudiantes o bajo la guía del docente para la construcción de conocimiento en el marco de un contexto social y cultural.

\section{DOCENTES, INSTITUCIONES DE EDUCACIÓN SUPERIOR Y EL RETO DE LAS TECNOLOGÍAS DE INFORMACIÓN Y COMUNICACIÓN APLICADAS A LA EDUCACIÓN}

El uso de las TIC en los procesos de enseñanza-aprendizaje ha enfrentado la resistencia al cambio por parte de algunos docentes, sea porque desconocen su potencial para dinamizar y enriquecer los procesos o, como lo manifiestan López y De Escoriaza (2011), carecen de los conocimientos técnicos, del tiempo o de los recursos físicos para incorporar las TIC a su quehacer pedagógico y didáctico. 
Esta incorporación de las TIC a la educación es un reto para el docente, porque implica nuevas prácticas de enseñanza-aprendizaje que privilegian el intercambio de información, el desarrollo de procesos de pensamiento, la comunicación, interacción y colaboración entre estudiantes y docentes, la visualización de la información y el descubrimiento, construcción y comprensión de nuevos conocimientos, en ambientes donde los estudiantes se mueven con mayor flexibilidad, libertad y autonomía, siendo protagonistas y productores de su propio aprendizaje (López Carrasco, 2013, pp. 100-104). Este contexto demanda por parte del docente, además de una nueva concepción de su labor, competencias tecnológicas que incluyen capacidades para navegar por Internet, utilizar herramientas de comunicación en línea en tiempo sincrónico y asincrónico, usar computadores y dispositivos de comunicación para facilitar el aprendizaje y orientar la búsqueda y análisis de información, y el desarrollo de competencias comunicativas para el espacio digital (López Carrasco, 2013). La necesidad de desarrollar estas competencias en los docentes es a su vez un reto para las instituciones de educación superior, que no solo involucra acciones de capacitación, sino también revisión de condiciones contractuales docentes y revisión del modelo educativo.

Si bien es cierto que las universidades han implementado plataformas digitales para innovar en el contexto educativo y responder a las exigencias legales, acordes con las legislaciones educativas de cada país, estas por sí solas no garantizan la integración de las TIC al modelo educativo de la institución y a las prácticas docentes en el aula. Esto en parte se debe a la aplicación de las estrategias y métodos pedagógicos y didácticos que no involucran el uso de TIC, por lo que existe el interrogante sobre la necesidad de "modificar el modelo de enseñanza universitario en su globalidad" (Moreira, 2008) o rediseñarlo para que incorpore las TIC a los procesos de enseñanza-aprendizaje, dentro de un modelo cuyas bases epistemológicas respondan a la nueva sociedad del conocimiento. Un error es mantener las prácticas educativas del viejo modelo, utilizando las TIC solo como un apoyo tecnológico a las mismas (López Carrasco, 2013, p. 96), mas no como medios dinamizadores del aprendizaje.

En este sentido y como lo afirma Carnoy (2004), la educación superior permanece anclada en procedimientos tradicionales que permean los procesos de enseñanza-aprendizaje, a pesar de que algunos docentes hacen esfuerzos para integrar las TIC a los mismos. Para que estos se sumen y generen la sinergia suficiente para facilitar y dinamizar el aprendizaje, es importante que cada institución actualice su modelo educativo integrando estas tecnologías a los procesos de enseñanza-aprendizaje, entrando a redefinir las interrelaciones entre estudiantes y docentes, los roles asumidos, los propósitos de formación y la evaluación.

Sobre cómo cambian las interrelaciones entre docentes y estudiantes, es importante reflexionar al respecto, por cuanto se pasa del ámbito tradicional donde los primeros poseían un saber para trasmitirlo y los segundos requerían adquirirlo (Palamidessi, 2006) a situaciones donde se integran las TIC a los procesos de enseñanza-aprendizaje, y se permite así a los estudiantes mayor libertad de exploración, observación y análisis 
de información; así mismo, se les conduce a experiencias significativas y se les empodera como constructores de su propio aprendizaje, en tanto que los docentes se convierten en diseñadores de las actividades de aprendizaje que integran estas tecnologías, constituyendonuevos espacios educativos acordes con la dinámica de la sociedad del conocimiento.

Este empoderamiento se hace posible debido a que un alto porcentaje de nuevos estudiantes universitarios ha crecido en esta época donde de manera continua emergen nuevas TIC que son utilizadas en los espacios sociales y laborales, por lo que cuentan con el conocimiento técnico sobre la manera como estas funcionan. Esta competencia personal habilita a los estudiantes aventajados en el uso cotidiano de las TIC, para que se conviertan en facilitadores del uso de las mismas, tanto para sus compañeros menos aventajados, como para sus docentes, lo que contribuye al desarrollo de relaciones horizontales y simétricas en los procesos de enseñanza-aprendizaje, conducentes a un aprendizaje colaborativo.

Ante estas nuevas circunstancias, es importante promover el desarrollo docente en el campo de las TIC (Tello y Aguaded, 2009) aplicadas a la educación, para lo cual se deben tener en cuenta las condiciones personales y los términos de vinculación de los docentes a las instituciones universitarias, por cuanto estas determinarán el grado de involucramiento de cada docente con el uso de estas en los procesos de enseñanzaaprendizaje. Entre las condiciones personales están el interés, la disposición y las aptitudes de cada docente frente al uso de estas tecnologías, y entre las condiciones de vinculación están los tipos de contratación y las funciones asignadas en el marco de la integración de las mismas a la educación. Por cuanto no todos los docentes tienen el interés, la disposición o las aptitudes para el uso de las tecnologías de información y comunicación, y hay aspectos contractuales y administrativos que no necesariamente contemplan el uso de las mismas en los procesos educativos, existen restricciones de índole personal o administrativo que obstaculizan la integración de las TIC a los procesos de enseñanza-aprendizaje.

Por esto se requiere capacitación, sensibilización y motivación de los docentes por parte de las instituciones universitarias, para que estos incorporen el uso de las TIC a su quehacer educativo (Salinas, 2004) y actualización de aspectos contractuales y administrativos, que faciliten la incorporación de las TIC a los procesos enseñanza-aprendizaje.

\section{Estudiantes, docentes y cambios en sus roles e interrelaciones}

Las TIC son consideradas por la comunidad académica como herramientas de apoyo para facilitar los aprendizajes cuya aplicación da lugar a cambios en la relación pedagógica entre estudiantes y docentes (Joao, 2002), lo que indica que es necesario reevaluar las prácticas educativas que se han venido aplicando en los procesos de enseñanza aprendizaje. Según Lau y Villanueva (s.f.), se deben consideran aspectos como la disponibilidad de información multimedia de uso interactivo para potenciar la transformación de la información en conocimiento. Esto permite experiencias de aprendizaje multisensoriales (Ministerio de Educación Nacional, 2013); la disponibilidad 
de múltiples fuentes, que facilita a los estudiantes la búsqueda, consulta, interpretación, interrelación y análisis de variedad de información; y el desarrollo de actividades de enseñanza-aprendizaje mediadas por las TIC que incrementan la creatividad e innovación (Ministerio de Educación Nacional, 2013).

Para que todo lo anterior sea posible, es importante identificar cuáles son las necesidades de formación de los docentes respecto al uso de las TIC como medio de apoyo y gestión de las prácticas educativas, para habilitar a los mismos en el diseño de actividades de aprendizaje, materiales de estudio y evaluaciones, que utilicen todo el potencial generador de aprendizaje de las TIC.

Como condición para aprovechar ese potencial de apoyo a la educación, se requiere primero hacer el tránsito de una educación centrada en la enseñanza donde el docente es el protagonista a una educación centrada en el aprendizaje, donde el protagonismo se traslada al estudiante. Dado este cambio, el rol del docente se transforma y se enfoca en el diseño y planeación de ambientes, medios y experiencias de aprendizaje; en el ejercicio como tutor, orientador y facilitador del aprendizaje y del uso de fuentes de información; y en la construcción y administración de redes de aprendizaje. En cuanto a la transmisión de contenidos, labor tradicionalmente ejercida por el docente, esta ya ha sido transferida en mayor grado a los recursos TIC y particularmente a la web, en forma de bases de datos, bibliotecas virtuales, páginas especializadas y repositorios de información, donde el estudiante puede encontrar múltiples datos e información bajo la guía del docente.
Otro aspecto por considerar es la interacción entre los estudiantes, parte importante del proceso enseñanza-aprendizaje, ya que aprenden unos de otros a través del intercambio de visiones y opiniones, y la conformación de grupos de trabajo colaborativo y comunidades de aprendizaje, lo cual genera ambientes y experiencias en las que todos construyen su aprendizaje a través del intercambio con los otros. Así, lo que cada estudiante aporta al curso durante estos espacios de trabajo colaborativo, foros y chats se convierte en valor agregado para el logro de los objetivos de aprendizaje (Martin, 1999, p. 208). Es decir que cada estudiante se convierte en facilitador y dinamizador de la construcción del conocimiento grupal, coordinador y constructor de redes de conocimiento.

Lo anterior es posible gracias a que existen diferentes niveles de desarrollo de competencias digitales entre los estudiantes, por lo que la significancia que para estos tiene el uso de las TIC varía, lo que hace más complejo el entramado de interrelaciones entre estudiantes y docentes, ya que el desarrollo de dichas competencias oscila entre los estudiantes que nacieron al tiempo con la Internet y han crecido a la par con los avances de las TIC, y los que han tenido que aprender con el paso del tiempo, por lo que las actitudes de cada quien frente al uso de estas variarán según las condiciones personales.

De los resultados de la investigación de Gutiérrez, Palacios y Torrego (2010, pp. 178, 179) se identificaron cuatro conglomerados de estudiantes universitarios, cada uno con sus propias actitudes: 
Figura 2. Conglomerados de estudiantes universitarios

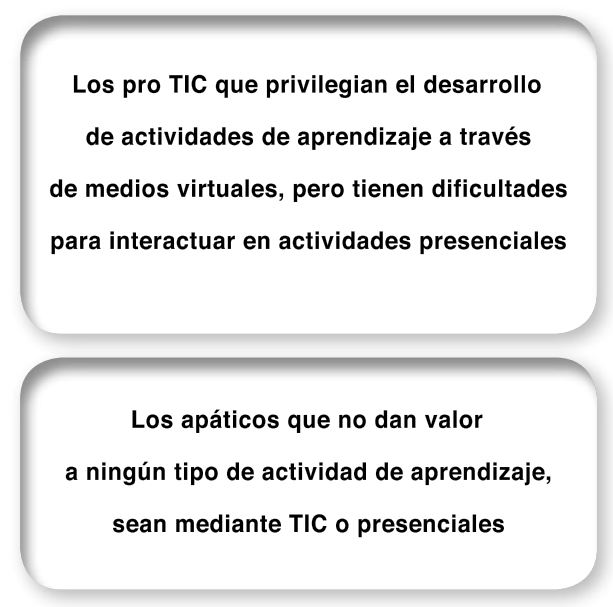

Esta diversidad de actitudes de los estudiantes representa mayores retos para los docentes, pues además de facilitadores de actividades y ambientes de aprendizaje, deben ser catalizadores de las expectativas de diferentes tipos de estudiantes frente a las TIC como medios de apoyo para la construcción de los aprendizajes, lo cual los convierte en administradores de esta diversidad, buscando una interrelación adecuada con cada grupo o tipo de estudiantes, en pro del logro de los objetivos de aprendizaje.

Así, el docente pasa de transmisor y fuente de información a mediador entre experiencias de aprendizaje, información y construcción de nuevo conocimiento (Joao, 2002), a través de actividades de aprendizaje mediadas por la TIC y combinadas con trabajos de articulación entre el conocimiento y la realidad, para una mejor comprensión de esta última (Ministerio de Educación Nacional Colombia, 2005). Por esta razón,el estudiante se convierte en un agente activo en la
Los anti TIC, que solo desarrollan

las actividades estrictamente necesarias

para aprobar las asignaturas

Los neutrales que privilegian las actividades

presenciales en el aula de clase, pues

consideran que aprenden más a través

del intercambio personal con compañeros

y docentes, aunque no rechazan las TIC

y reconocen su utilidad para consulta de

información y presentación de evaluaciones.

búsqueda, administración e interpretación de información (Joao, 2002) y construcción de su aprendizaje.

Para que los docentes estén en capacidad de asumir estos nuevos roles, se requiere una formación para estos que garantice el desarrollo de sus competencias para diseñar estrategias de aprendizaje que involucren el uso y aplicación de herramientas TIC para el logro de los objetivos de aprendizaje (Ministerio de Educación Nacional Colombia, 2005). Ejemplo de estas estrategias son el aprendizaje basado en problemas o los equipos de aprendizaje, donde el docente se convierte en orientador y facilitador de aprendizajes (Ministerio de Educación Nacional Colombia, 2005) que se pueden articular con herramientas de las TIC, para fortalecer los procesos de aprendizaje.

De acuerdo con la Unesco la misión docente consiste en ayudar al estudiante a adquirir capacidades en el uso de TIC y diseñar 
oportunidades y entornos de aprendizaje en el aula, que faciliten el uso de las mismas para aprender. En este sentido y según Salinas (2004), los docentes son actores esenciales en el sistema educativo e imprescindibles para la realización de los cambios en los procesos enseñanza-aprendizaje, derivados de la aplicación y uso de las TIC, por lo cual es necesario que las instituciones educativas brinden a sus docentes la oportunidad de formarse para alcanzar el conocimiento y dominio del potencial de las tecnologías, interactuar con la comunidad educativa y social y responder a las necesidades de formación de la sociedad (Salinas, 2004).

Así es como la integración de las TIC a los procesos de enseñanza-aprendizaje influye en el cambio de las funciones y el papel del docente, y entendida su actividad como un todo, incluye también en el diseño de experiencias de aprendizaje y el fomento de los procesos de reflexión y construcción del conocimiento por parte de los estudiantes, haciendo uso de ambientes virtuales de aprendizaje, provistos por la institución educativa, como apoyo a la labor docente.

Como las TIC apoyan el desarrollo de los procesos de enseñanza y aprendizaje poniendo a disposición de los estudiantes diversas alternativas para conseguir de manera sencilla los objetivos de aprendizaje (Rodriguez Cobos, 2009), les permite convertirse en protagonistas de su propio aprendizaje, y el rol del docente evoluciona desde transmisor de información a facilitador de los procesos de aprendizaje, orientador de las actividades de aprendizaje y administrador de los ambientes virtuales de aprendizaje, lo que cambia la dinámica tradicional de la interrelación entre estudiante y docente.
También el docente avanza hacia el uso de medios informáticos en los cuales almacena, procesa y difunde toda la información que el alumno necesita en su proceso de formación (Rodríguez Cobos, 2009) y construcción de su aprendizaje. Esta situación aumentan la interacciones entre estudiantes y docentes, y genera espacios propicios para un aprendizaje significativo (Rodríguez Cobos, 2009) y colaborativo.

Por esto es necesario que el docente analice cuidadosamente las situaciones de enseñanza-aprendizaje, identifique cuáles son los objetivos de aprendizaje del curso o asignatura por orientar, formule procedimientos de retroalimentación del aprendizaje y evaluación del logro de los objetivos de aprendizaje y responda dos preguntas: ¿cuáles son las actividades de aprendizaje que los estudiantes desarrollan actualmente? y ¿cuáles son las actividades de enseñanza que permitirán que el aprendizaje significativo ocurra? (Fink, 2003, p. 103). Las respuestas a estas dos preguntas se requieren tanto para actividades presenciales, como para actividades mediadas por TIC, puesto que el diseño de cualquiera de estas categorías debe responder a los objetivos de aprendizaje buscados. Y es en este caso donde el docente ejerce los roles de arquitecto y gestor de experiencias y ambientes de aprendizaje.

En una clase tradicional el docente presenta su comprensión de los temas de estudio y el estudiante escucha, toma notas, hace lecturas y eventualmente formula preguntas y participa en discusiones en clase. Pero varias investigaciones demuestran que los estudiantes aprenden más e incorporan el conocimiento por mayor tiempo, mediante el uso de métodos activos de aprendizaje, 
donde el estudiante desarrolla actividades y reflexiona sobre estas (Fink, 2003, p. 103), para identificar lo que ha aprendido y como lo ha aprendido, sea de forma individual o grupal (Fink, 2003, pp. 104).Por su parte el docente desempeña el rol de orientador del proceso reflexivo del estudiante, en tanto que este se apropia de su propio aprendizaje.

Resumiendo, al hacer el docente uso de las TIC como instrumentos de apoyo y complemento para los procesos de enseñanzaaprendizaje en el aula, ejerce los roles de diseñador de ambientes de aprendizaje, administrador de esos ambientes, usuario de estas tecnologías y orientador del proceso de aprendizaje. Para poder ejercer estos roles acertadamente, requerirá aprender a desarrollar materiales en línea para los cursos, utilizar los instrumentos de comunicación con los estudiantes que tiene la plataforma y diseñar evaluaciones a través de la misma.

De manera similar, en actividades de aprendizaje mediadas por las TIC la interacción entre los mismos estudiantes se convierte en una parte importante del proceso enseñanza-aprendizaje, ya que estos aprenden unos de otros, a través de la conformación de grupos de trabajo colaborativo y comunidades de aprendizaje donde se construye el aprendizaje a través del intercambio con los otros (Martin, 1999, p. 208). Así los estudiantes asumen un rol más activo el cual necesita que estén motivados (Martin, 1999, p. 204) y que tengan la voluntad de aprender (Diaz y Hernández, 2010, p.57); es decir, contar con la disposición o interés de los estudiantes de aprender y darle sentido a lo que aprenden (Ausubel, Novaky Hasenian, 1983, p. 50).
En conclusión, la integración de las TIC a los procesos de enseñanza-aprendizaje en la educación superior contribuye al desarrollo del aprendizaje tanto en estudiantes, como docentes, pues si bien es cierto que en este contexto la educación es centrada en el aprendizaje del estudiante, no es menos cierto que los docentes a través de procesos de formación y cambio de roles en los procesos de enseñanza-aprendizaje, que hacen uso de TIC, desarrollan a su vez su propio aprendizaje respecto a estas. Como el estudiante se convierte en responsable y constructor de su propio aprendizaje y dinamizador de la construcción del conocimiento grupal que emerge en los grupos de trabajo colaborativo y redes de estudio, el rol del docente necesariamente transita hacia orientador, facilitador y guía de los estudiantes hacia la construcción de su aprendizaje.

Como consideración a lo presentado hasta este punto, las interrelaciones entre docentes y estudiantes se ajustan como resultado de la integración de las TIC a las actividades de enseñanza-aprendizaje, y se convierten en interrelaciones horizontales y multidireccionales propias del trabajo colaborativo y el aprender con otros, en contraposición a las interrelaciones jerárquicas, verticales y unidireccionales de la transmisión de conocimientos hecha por el docente. De otra forma, como lo afirma Gutiérrez, Palacios y Torrego (2010, p. 175) "una integración curricular de las TIC sin la necesaria reflexión desde postulados educativos nos llevaría a convertirlas en refuerzo de un modelo reproductor unidireccional y vertical de la enseñanza como transmisión de contenidos"; es decir, se estarían tecnificando prácticas educativas tradicionales donde se privilegia la educación centrada en la enseñanza y 
el docente como protagonista, en contravía con los aprendizajes significativos y colaborativos, donde la educación es centrada en el estudiante, con lo cual las TIC no aportarían al aprendizaje. Por esta razón, es necesario capacitar a los docentes no solo en el uso de las TIC aplicadas a la educación-las cuales comprenden según Johnson, Adams, y Cummins (2012) tecnologías en educación a corto y mediano plazo que van desde aplicaciones para móviles, hasta impresiones en tercera dimensión-, sino también en estrategias formales de aprendizaje, que sean acordes con el uso de las TIC, entre las que se encuentran el aprendizaje basado en problemas o los equipos de aprendizaje, que se presentan en la siguiente sección.

\section{EJEMPLOS DE ESTRATEGIAS DE ENSEÑANZA-APRENDIZAJE CON APLICACIÓN DE LAS TECNOLOGÍAS DE INFORMACIÓN Y COMUNICACIÓN PARA PROGRAMAS PRESENCIALES DE EDUCACIÓN SUPERIOR}

\section{Aprendizaje basado en problemas}

El aprendizaje basado en problemas (ABP) es una estrategia de enseñanza-aprendizaje (Diazy Hernández, 2010) que se basa en el diseño de actividades de aprendizaje donde se presentan problemas propios de la vida real, con el objetivo de promover aprendizajes situados, enfrentando a los estudiantes a experiencias que les permitan desarrollar competencias similares o iguales a las que encontrarán en situaciones de la vida profesional. Esta estrategia responde al aprendizaje significativo, partiendo del conocimiento, experiencias previas y motivaciones del estudiante, para a partir de estos construir su nuevo aprendizaje. El aprendizaje basado en problemas ABP es de amplio uso en educación superior y propicia el trabajo colaborativo. Según Torp y Sage (1998), el ABP tiene tres características centrales: organiza las actividades de aprendizaje alrededor de problemas relevantes; convierte a los estudiantes en actores de las problemáticas de estudio; genera un ambiente de aprendizaje donde los estudiantes desarrollan actividades cognitivas complejas como solución de problemas y toma de decisiones, y hacen trabajo colaborativo, en tanto que el docente orienta y apoya los procesos de búsqueda de información y análisis.

En general, las estrategias y actividades de resolución de problemas en el ámbito educativo presentan a docentes y estudiantes la oportunidad de establecer actividades de forma guiada que van desde identificar información y aplicarla en la solución de un problema hasta la organización de tareas, elaboración de diagnósticos y presentación de informes en un contexto más complejo. Se busca así fomentar las capacidades de pensamiento, el análisis crítico y desarrollar en los estudiantes habilidades sociales que les permitan presentar sus puntos de vista y aceptar los de los demás.

El ABP a su vez enfatiza en el autoaprendizaje y la autoformación. En ABP se fomenta la autonomía cognoscitiva, ya que se enseña y aprende a partir de problemas que tienen significado para los estudiantes, se utiliza el error como una oportunidad más para aprender y se le otorga un valor importante a la autoevaluación y la evaluación formativa, cualitativa e individualizada (Dueñas, 2001). Entonces el ABP se traduce en un proceso de indagación realizado por 
el estudiante que busca resolver preguntas, dudas e incertidumbres sobre fenómenos o problemas complejos (Barell, 1999, p. 21). Identifica problemas, plantea interrogantes, investiga, propone soluciones, las documenta y socializa en grupo; esto da origen a la formación de una comunidad de aprendizaje, donde los participantes se "escuchan entre sí, están abiertos a diferentes puntos de vista y pueden trabajar en colaboración" (Barell, 1999, p. 21), para obtener respuestas a los interrogantes y soluciones a los problemas estudiados.

Así visto, el aprendizaje basado en problemas como estrategia de aprendizaje genera el aprendizaje con base en redes de estudiantes; parte de la información como fuente de aprendizaje; tiene como punto de inicio el conocimiento que ya trae el estudiante y como punto de llegada el conocimiento construido a través del estudio de la problemática planteada; y se apoya en las TIC para la búsqueda de nueva información (Joao, 2002) que será analizada para la búsqueda de una solución al problema estudiado. Es una estrategia de aprendizaje centrada en el estudiante, donde este ejerce un papel activo en la construcción de su conocimiento, en tanto que el docente ejerce el papel de facilitador y orientador de esta experiencia de aprendizaje, por lo que es aplicable a ambientes de aprendizaje, presenciales y mediados por TIC.

\section{Equipos de APRENDIZAJE}

Dado que el aprendizaje visto desde el socioconstructivismo es un proceso social que se dinamiza a través de las interrelaciones con otros, surge el concepto del aprendizaje colaborativo, como forma de organización para el aprendizaje en pequeños grupos constituidos por máximo cinco integrantes (Ferreiro, 2013), donde las interacciones entre pares desarrollan habilidades, actitudes y valores que permiten la construcción del nuevo aprendizaje para todos sus integrantes.

Por esto, la estrategia de equipos de aprendizaje utiliza de forma sistemática el trabajo en pequeños grupos, desarrollado a través de una secuencia planeada de actividades (Fink, 2003, p.132). El trabajo colaborativo genera estados de ánimo conducentes al aprendizaje eficaz de los integrantes y al desarrollo de la competitividad del equipo (Ferreiro, 2013).El proceso inicia con la asignación de un tema sobre el cual cada estudiante hace su búsqueda, análisis y síntesis de información obtenida tanto en el mundo real como en el virtual. En clase se presenta una evaluación sobre los contenidos del tema, de forma individual o colectiva, es decir, por grupos (Fink, 2003, p. 122). Estas actividades conducen al estudiante a un nivel moderado de comprensión de los contenidos. Luego los estudiantes se reúnen en pequeños grupos para aprender cómo aplicar esos contenidos a una serie de ejercicios prácticos (Fink, 2003, p. 122) y posteriormente presentan una prueba que valora la comprensión de contenidos y la habilidad para aplicar estos a situaciones similares a la realidad. Este ciclo se repite a lo largo del curso, con lo que los grupos se cohesionan, a medida que avanza el tiempo, se constituyen en equipos de aprendizaje, capaces de realizar actividades de aprendizaje cada vez de mayor nivel (Fink, 2003, p. 122).

Las actividades para el desarrollo de esta estrategia hacen uso de espacios presenciales y virtuales, pero tanto en unos como en otros emergen el trabajo colaborativo, para 
el logro de los objetivos de aprendizaje individuales y del grupo. Es de observar que la búsqueda de análisis y síntesis inicial de información es una actividad que se basa en el uso de las TIC. Igual sucede con el trabajo en pequeños grupos, el cual puede apoyarse en recursos TIC para las comunicaciones entre los integrantes, y recursos para el trabajo colaborativo como herramientas para construcción de documentos en tiempo real, seguimiento del cumplimento de tareas y trabajo simultáneo en tiempo real.

\section{Reflexiones finales}

Luego de la reflexión acerca de cómo influyen las TIC en los roles y las interrelaciones entre estudiantes y docentes en los procesos de enseñanza-aprendizaje en programas presenciales de educación superior, se concluye que la nueva dinámica de los procesos de enseñanza-aprendizaje mediados por TIC ha conducido a la reevaluación de los roles de estudiantes y docentes. El estudiante se convierte en responsable y constructor de su propio aprendizaje y dinamizador de la construcción del conocimiento grupal cuando este emerge en los grupos de trabajo colaborativo y redes de estudio. Por su parte, el docente asume el rol de arquitecto y gestor de ambientes y experiencias de aprendizaje, y orientador, facilitador y guía de los estudiantes hacia la construcción de su aprendizaje.

Estos cambios de roles tradicionales de estudiantes y docentes van acompañados de cambios en la dinámica de las interrelaciones entre estos actores, las cuales pasan de verticales y jerárquicas, a horizontales y cooperativas. Esto se debe a que en este nuevo contexto de la sociedad del conocimiento, el docente deja de ser transmisor de la información, para convertirse en guía y orientador de la búsqueda, comparación y análisis de la misma para la construcción de aprendizaje, asumiendo la web la función de transmisión de información, la cual está a disposición de los estudiantes.

Por esto es importante desarrollar actividades de aprendizaje que integren tecnologías de información y comunicación, y permitan a los estudiantes descubrir el significado de la nueva información e identificar la manera de alcanzar y regular su propio proceso de aprendizaje. Se espera también que la utilización de ambientes virtuales propicien el desarrollo y gestión de estrategias para mantener registrada la información, relacionar la nueva información con los conocimientos previos, fomentar el pensamiento crítico y la creatividad, y motivar y generar actitudes para que el estudiante pueda convertirse "en el responsable de su propio proceso de aprendizaje" (Villalobos, 2006, p. 213).

Otro aspecto relevante de la influencia de las TIC en los procesos de enseñanza aprendizaje se refiere a la capacidad que tienen estas de estimular el aprendizaje significativo en cada estudiante, el trabajo colaborativo manifestado a través de las comunicaciones entre estudiantes y entre estos y docentes, y el aprendizaje colaborativo que se traduce en un constructivismo social del aprendizaje, a través de grupos de trabajo y redes de aprendizaje posibles gracias al apoyo de las herramientas TIC y el acceso a la web.

Las acciones de trabajo grupal dependen de muchos factores y de las interrelaciones que se dan entre los estudiantes, por lo que la adecuada selección de la estrategia de 
aprendizaje facilitará la integración de estos para lograr un aprendizaje en colaboración, que demanda por parte del docente mayor capacitación y compromiso en la planificación de las actividades, seguimiento y evaluación, amén de la habilidad para orientar adecuadamente el desarrollo de habilidades interpersonales de los estudiantes.

Aunque la inclusión de las TIC en la educación superior no garantiza por sí sola la construcción colectiva de conocimiento, ya que como lo expresa Hine (2004) "el agente de cambio no es la tecnología en sí misma, sino los usos y la construcción de sentido alrededor de ella", su influencia es tal que se hace necesario repensar el diseño y aplicación de estrategias de enseñanza y aprendizaje en ambientes virtuales que promuevan este aprendizaje colaborativo para el desarrollo de competencias en concordancia con los nuevos retos de la sociedad del conocimiento.

Pero para que todo lo expuesto sea posible, es necesario cambiar la formación docente, no solo en conocimientos sobre TIC, sino en cuanto a motivación y sensibilización frente a la capacidad de aporte de las TIC al logro de los objetivos de aprendizaje. La capacitación en estrategias y herramientas de integración de las TIC en los procesos de enseñanza y aprendizaje no son suficientes; se requiere promover un cambio de actitud de los docentes y en este sentido, como lo mencionan Chocarro, González-Torres y Sobrino (2007, p. 93)“al igual que con los alumnos, los profesores deben percibir la utilidad de su aprendizaje y, para ello, es necesario que la formación sea auténtica y orientada a la práctica, de modo que puedan analizar en su contexto (de modo situado) la relación entre la enseñanza y el aprendizaje".
En relación con los estudiantes, es importante identificar sus actitudes respecto al uso de las TIC en los procesos de enseñanza-aprendizaje. Según Sobrino (2011, p. 131) “la utilización estratégica de las TIC para el aprendizaje escolar no se deriva de la destreza en su utilización como medio de comunicación en las redes sociales"; es decir, que el hecho de contar con estudiantes conocedores y usuarios de las TIC en sus espacios personales y sociales (nativos digitales) no asegura el éxito de estrategias de aprendizaje mediadas por TIC. Por esto la necesidad de aplicar estrategias de enseñanza-aprendizaje que potencialicen el trabajo colaborativo y la construcción de conocimiento, mediante el uso de TIC.

En conclusión, las TIC al ser aplicadas a los procesos de enseñanza aprendizaje no solo generan cambios en los roles e interrelaciones entre estudiantes y docentes, sino que promueven el emerger de una nueva cultura educativa, donde el transmisor de conocimiento es la web, el aprendizaje se construye a través de la interacción con las TIC y con los otros, estudiantes o docentes, los estudiantes son constructores de su aprendizaje y los docentes constructores de ambientes y experiencias de aprendizaje y todos aprenden de todos en un ambiente de constructivismo social.

\section{REFERENCIAS}

Ausubel, D., Novak, J. y Hasenian, H. (1983). Psicología Educativa. México: Trillas.

Barell, J. (1999). El aprendizaje basado en problemas. Buenos Aires: Ediciones Manantial SRL.

Barkley, E., Cross, P. y Howell. (2007). Técnicas de aprendizaje colaborativo: manual para el profesorado universitario. Madrid: Ediciones Morata S.L. 
Carnoy, M. (2004). Las TIC en la enseñanza. Posibilidades y retos. Recuperado el 9 de Octubre de 2013, de http://www.uoc.edu/ inaugural04/esp/index_content.html

Cerezo Huertas, H. (julio-diciembre de 2006). Corrientes pedagógicas contemporáneas. Revista electrónica de pedagogía.4, (7), 1-19.

Chocarro, E., González-Torres, M. y Sobrino, A. (2007). Nuevas orientaciones en la formación del profesorado para una enseñanza centrada en la promoción del aprendizaje autorregulado. Estudios sobre educación, (12), 81-98.

Diaz Barriga, F. y Hernández, G. (2010). Estrategias docentes para un aprendizaje significativo. México: McGraw-Hill.

Dueñas, V. H. (2001). El aprendizaje basado en problemas como enfoque pedagógico en la educación en salud. Colombia Médica, 32(4), 189-196. Recuperado de http://redalyc.uaemex.mx/redalyc/ pdf/283/28332407.pdf

Ferreiro Gavrie, R. (30 de septiembre de 2013). Del aprendizaje grupal al cooperativo. Recuperado el 9 de octubre de 2013, de http://www.redtalento.com/articulos/ website\%20revista\%20magister\%20articulo\%201.pdf

Fink, L. D. (2003). Creating significant learning experiences. San Francisco: John Wiley \& Sons Inc.

Gutierrez, A., Palacios, A. y Torrego, L. (2010). Tribus digitales en las aulas universitarias. Revista Científica de Educomunicación, XVII(34), 173-181.

Hine, C. (2004).Etnografía virtual[versión electrónica]. Barcelona: Editorial Luoc.

Joao, O. P. (2002). Pedagogía informacional: enseñar a aprender en la sociedad del conocimiento.Universitat Oberta de Catalunya. recuperadod de http://www.uoc. edu/web/esp/art/uoc/opicardo0602/ opicardo0602.html
Johnson, L., Adams, S. y Cummins, M. (2012). Informe Horizon del NMC: edición para la enseñanza universitaria 2012.Austin: The New Media Consortium.

Johnson, D. y Johnson, R. (1999). Aprender juntosysolos:aprendizajecooperativo,competitivo e individualista. Argentina: Aique Grupo Editor S.A.

Lau Sánchez, N. y Villanueva Salazar, L. (2010, noviembre). Las personas adultas y la apropiación de las TIC'S: ¿aceptación o rechazo? III Congreso Internacional de Gerontología. Universidad de Costa Rica, San José, Costa Rica.

López Carrasco, M. A. (2013). Aprendizaje, competencias y TIC. México: Pearson.

López, J. G. y De Escoriaza, J. C. (2011). El pensamiento docente y su influencia en la implantación de las tecnologías de la información y la comunicación en el aula: desafíos y oportunidades. Contextos educativos: Revista de Educación, 67-84.

Márques, P. (1999). Concepciones sobre el aprendizaje. Recuperado el 10 de octubre de 2012, de http://www.peremarques.net/ aprendiz.htm

Martin, C. (1999). Las 7 cibertendencias del siglo XXI. Bogotá: McGraw-Hill.

Ministerio de Educación Nacional (2013). Competencias TIC para el desarrollo profesional docente. Bogotá: Oficina de Innovación Educativa con Uso de Nuevas Tecnologías.

Ministerio de Educación Nacional Colombia. (febrero-marzo de 2005). Uso pedagógico de tecnologías y medios de comunicación. Exigencia constante para docentes y estudiantes. Altablero, pp. 3-5. Recuperado de http://www.mineducacion.gov. co/1621/article-87580.html

Moreira, M. A. (septiembre de 2008). Innovación pedagógica con TIC y el desarrollo 
de las competencias informacionales y digitales. Investigación en la escuela (64), 5-18.

Ontoria, A. y Ballesteros, A. (2011). Mapas conceptuales. Una técnica para aprender. Madrid: Narcea S.A. de ediciones.

Palamidessi, M. (2006). La escuela en la sociedad de redes. Una introducción a las tecnologías de la informática y la comunicación en la educación. Buenos Aires: Fondo de Cultura Económica.

Rodriguez Cobos, E. M. (noviembre de 2009). Ventajas e inconvenientes de las TIC en el aula. Cuadernos de Educación y Desarrollo, 1(9). Recuperado el 10 de agosto de 2014, de http://www.eumed.net/rev/ced/09/ emrc.htm

Salinas, J. (2004). Innovación docente y uso de las TIC en la enseñanza universitaria. Revista Universidad y Sociedad del Conocimiento, XIV(33), 1-16.

Serrano, J. M. y Pons, R. M. (2011). El constructivismo hoy: enfoques constructivistas en educación. Revista Electrónica de Investigación Educativa, 13(1). Recuperadoel 10 de agosto de 2014, de http:// redie.uabc.mx/vol13no1/contenido-serranopons.html

Servicios Educativos del Magisterio. (2003). Modelos educativos, pedagógicos y didácticos (2da. edición) (Vol. II). Bogotá: Ediciones S.E.M.
Sobrino, A. (2011). Proceso de enseñanzaaprendizaje y web 2.0. Análisis del conectivismo como teoría del aprendizaje post-constructivista. Estudios sobre educación(20), 117-140.

Tello Díaz, J. y Aguaded Gómez, J. I. (2009). Desarrollo profesional docente ante los nuevos retos de las tecnologías de la información y comunicación en los centros docentes educativos. Revista de medios y educación, 31-47.

Tobón, M. I. (2007). Diseño instruccional en un entorno de aprendizaje abierto. Pereira: Universidad Tecnológica de Pereira.

Torp, L. y Sage, S. (1998). El aprendizaje basado en problemas. Argentina: Amorrortu.

Unesco. (2008). Estándares Unesco de competencia en tic para docentes. Recuperado de http://www.eduteka.org/EstandaresDocentesUnesco.php

Unesco. (s.f.). Unesco estandares docentes. Recuperado el 30 de septiembre de 2013, de http://www.eduteka.org/pdfdir/UNESCOEstandaresDocentes.pdf

Villalobos, E. M. (2006). Didáctica integrativa y el proceso de aprendizaje. México D.F.: Editorial Trillas 